Check for updates

Cite this: Phys. Chem. Chem. Phys. 2019, 21, 1375

Received 29th October 2018 , Accepted 18th December 2018

DOI: $10.1039 / c 8 c p 06716 c$

rsc.li/pccp

\title{
Kinetic Monte Carlo simulations of organic ferroelectrics $\dagger$
}

\author{
Tim D. Cornelissen, (D) ${ }^{a}$ Michal Biler, (D) ${ }^{b}$ Indre Urbanaviciute, (D) ${ }^{a}$ \\ Patrick Norman, (D) ${ }^{\mathrm{b}}$ Mathieu Linares (D) ${ }^{\mathrm{bc}}$ and Martijn Kemerink (D) *a
}

\begin{abstract}
Ferroelectrics find broad applications, e.g. in non-volatile memories, but the switching kinetics in real, disordered, materials is still incompletely understood. Here, we develop an electrostatic model to study ferroelectric switching using 3D Monte Carlo simulations. We apply this model to the prototypical small molecular ferroelectric trialkylbenzene-1,3,5-tricarboxamide (BTA) and find good agreement between the Monte Carlo simulations, experiments, and molecular dynamics studies. Since the model lacks any explicit steric effects, we conclude that these are of minor importance. While the material is shown to have a frustrated antiferroelectric ground state, it behaves as a normal ferroelectric under practical conditions due to the large energy barrier for switching that prevents the material from reaching its ground state after poling. We find that field-driven polarization reversal and spontaneous depolarization have orders of magnitude different switching kinetics. For the former, which determines the coercive field and is relevant for data writing, nucleation occurs at the electrodes, whereas for the latter, which governs data retention, nucleation occurs at disorder-induced defects. As a result, by reducing the disorder in the system, the polarization retention time can be increased dramatically while the coercive field remains unchanged.
\end{abstract}

\section{Introduction}

Ferroelectric materials find application in a broad range of fields, but a full understanding of the switching and especially the depolarization kinetics on all length and time scales is still lacking. A variety of computational models have been employed to tackle this problem and study different aspects of ferroelectrics. ${ }^{1}$ First-principles calculations based on Density Functional Theory (DFT) are computationally expensive but can give detailed insight into phase diagrams, static domain structures or ultrathin films. ${ }^{2-5}$ Molecular dynamics (MD) simulations can predict domain features and kinetics on intermediate length and time scales. ${ }^{6,7}$ Finally, there are Monte Carlo simulations that are mainly based on electrostatic interactions. ${ }^{8-10}$ These simulations can address experimental length and time scales but are often restricted to idealized morphologies. In contrast, we develop here a 3D electrostatic model that can reproduce ferroelectric properties

\footnotetext{
${ }^{a}$ Complex Materials and Devices, Department of Physics, Chemistry and Biology (IFM), Linköping University, 58183 Linköping, Sweden.

E-mail: martijn.kemerink@liu.se

${ }^{b}$ Department of Theoretical Chemistry and Biology, School of Engineering Sciences in Chemistry, Biotechnology and Health, KTH Royal Institute of Technology, 10691 Stockholm, Sweden

'Swedish e-Science Research Centre (SeRC), KTH Royal Institute of Technology, 10450 Stockholm, Sweden

$\dagger$ Electronic supplementary information (ESI) available. See DOI: 10.1039/c8cp06716c
}

and kinetics on experimental time scales taking realistic, disordered morphologies as input.

The field of ferroelectrics has for long been dominated by inorganic materials such as barium titanate (BTO) and lead zirconate titanate (PZT), with the notable exception of the polyvinylidene fluoride polymer (PVDF) and its various copolymers. Recently, a new class of organic ferroelectrics has been explored, the small molecular liquid crystals. Whereas there have been numerous experimental works on the ferroelectric behavior of these materials, ${ }^{11,12}$ there is only a basic understanding of the underlying processes and kinetics.

As a prototype small molecular ferroelectric, we focus here on trialkylbenzene-1,3,5-tricarboxamide (BTA). Although BTA has received extensive interest for its self-assembly properties, ${ }^{13}$ it has only recently been experimentally proven to be ferroelectric. ${ }^{14-16}$ Most previous theoretical work on this material has thus been focused on the self-assembly properties and the dipole moment of single stacks. ${ }^{17-20}$ Recently, Zehe et al. used a simple 2D Ising model to investigate the geometrical frustration between the hexagonally packed columns and the different roles of nearest and next-nearest neighbor interactions on the nature of the ground state. ${ }^{21}$

Here we develop an electrostatic model that takes the full 3D morphology of BTA into account and reproduces experimentally observed ferroelectric properties using kinetic Monte Carlo simulations. We examine polarization hysteresis loops and retention, 
and we can rationalize the obtained parameter dependencies in the framework of thermally activated nucleation limited switching. We find an antiferroelectric ground state that in practice is not reached due to high activation energies leading to extremely slow depolarization kinetics. Structural disorder is found to be a critical parameter for polarization retention. The results show good agreement with experiments, and not only provide detailed insight into the mechanism of polarization switching in organic ferroelectrics, but also concrete guidelines to further improve the performance of organic, and possibly inorganic, ferroelectric devices.

\section{Model}

The molecular structure of BTA is shown in Fig. 1. It consists of a benzene core to which three chains are attached composed of a dipolar amide group and a flexible alkyl tail whose length can vary. Driven by $\pi-\pi$ stacking interactions between the benzene core and the formation of hydrogen bonds between the amide groups, these discotic molecules self-organize into supramolecular columnar structures (Fig. 1(c)). In each column, the dipolar amide groups form a triple helix, which results in a macrodipole that is oriented along the column axis. In the liquid crystalline phase, the columns organize in a hexagonal packing (Fig. 1(d)). Due to the flexibility of the alkyl tails, the side chains have enough mobility to allow rotation of the dipoles under an applied electric field. This flips the macrodipoles of the columns, and thereby the polarization of the material. In a recent paper we established experimentally that the bistable polarization of BTA reflects true ferroelectricity. ${ }^{14}$

The ferroelectricity in BTA is thus caused by the collective behavior of the dipolar amide groups. We therefore focus on these dipoles and their interactions. The dipoles have fixed positions given by the structure of the BTA molecule and the morphology of the system. We assume that the permanent dipoles $\overrightarrow{\mu_{0}}$ only have two possible directions, up and down, which differ only in the z-component of their dipole moment. On top of these permanent dipoles we have to take into account induced dipoles. These are the result of the electron clouds that get shifted by the net local electric field $\overrightarrow{E_{\mathrm{loc}}}$. They are directed along the local field: $\overrightarrow{\mu_{\text {ind }}}=\alpha \times \overrightarrow{E_{\mathrm{loc}}}$, with $\alpha$ the linear polarizability.
The total dipole is the sum of the permanent and induced dipoles, $\overrightarrow{\mu_{\mathrm{tot}}}=\overrightarrow{\mu_{0}}+\overrightarrow{\mu_{\mathrm{ind}}}$.

To determine the flipping probability of a dipole, we calculate the energy difference between the initial and final state based on the dipole-dipole interactions between all dipoles within a certain cutoff radius, as well as the interaction with the externally applied field. Interactions outside of the cut-off radius are taken into account using the reaction field method. ${ }^{22}$ Hence, the energy difference between the two possible polarization states of each dipole depends on the orientation of all other dipoles within the cutoff and has to be updated after each flipping event. We do not consider any rotational energy barrier between the two states as doing so would only add an offset to all energies involved and as such be equivalent to a change in the flipping rate prefactor. This prefactor determines the time scale of the simulations and changing it only results in absolute time/frequency differences in the results. The flipping rates are the input for a kinetic Monte Carlo simulation (kMC), which allows us to observe the dynamical behavior of the complete system and its response to externally applied fields. More details on all energy calculations and the simulation algorithm can be found in the ESI. $\dagger$

Being a liquid crystalline material, the system is subject to positional disorder, see Fig. 1(d). Based on previous experimental work, ${ }^{14,23,24}$ we implement this disorder by introducing defects that represent a break in the hydrogen-bonded triple helix. This divides a column into subcolumns, each with their own translational offset, rotational orientation, and handedness. We start with a fixed amount of disorder, but in the section "The effect of disorder" we will vary the amount of disorder and investigate how this influences the ferroelectric properties. Further details about the disorder parameters are given in Table S1 and Fig. S3 (ESI $\dagger$ ). We would like to note that this disorder is the only free parameter in the simulations. All other parameters are fixed and taken from experiment or DFT calculations, as discussed below and in the ESI. $\dagger$

\section{Results and discussion}

\section{Hysteresis loops}

The main characteristic of a ferroelectric material is its polarization hysteresis loop as a function of the applied electric field.

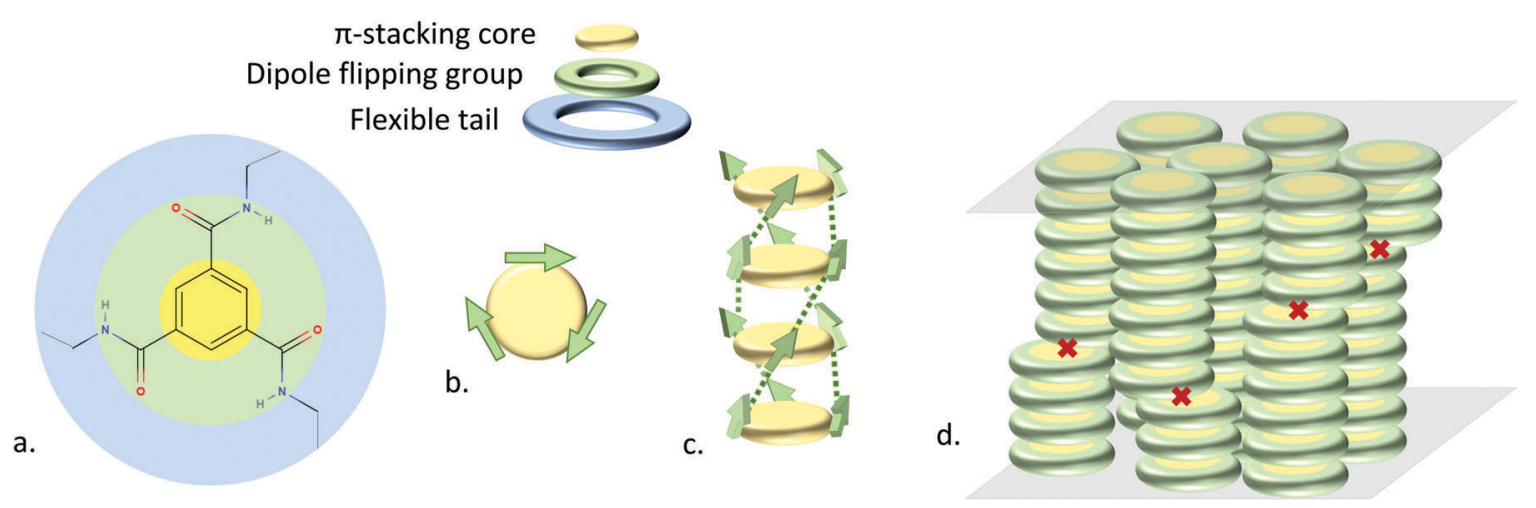

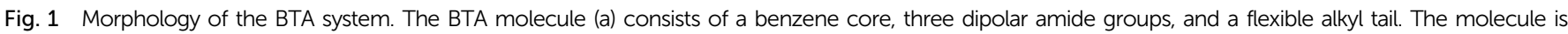

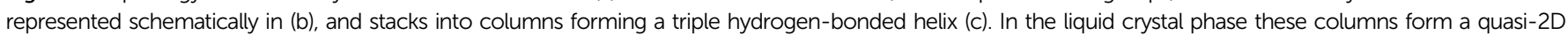

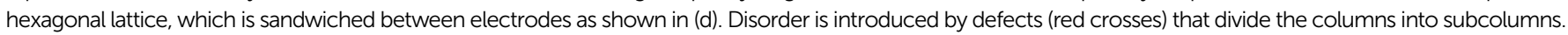


An example of a simulated hysteresis loop is shown in Fig. 2(a), with a shape that is typical for ferroelectrics. It should be noted that the loop is corrected for a linear background, as is done for the experimental loop in Fig. 2(a), as discussed in the ESI. $\dagger$ We will discuss three features of the hysteresis loops: (saturation) polarization, shape and coercive field.

First, the polarization of the system is simply the dipole density and is thus determined by the position, orientation and magnitude of all dipoles. Since our morphology is fixed, the polarization is governed by the permanent dipole $\mu$ and the polarizability $\alpha$. We determine these parameters using the results of DFT calculations on BTA columns. ${ }^{17,20,21}$ These calculations have shown that when forming columns, BTA molecules exhibit a cooperativity effect, meaning that the dipole moment per molecule increases when more molecules are added to the column. Typically, the dipole moment per molecule will increase from $\sim 7 \mathrm{D}$ to $\sim 12 \mathrm{D}$ upon column elongation from 2 to $>8$ BTA units. We thus have a permanent dipole of $7 \mathrm{D}$ per molecule, and an induced dipole of $5 \mathrm{D}$. Taking $\mu_{0}=4 \mathrm{D}$ per amide group and $\alpha=1 \mathrm{e}^{2} \mathrm{~V}^{-1}$ gives a good approximation with a permanent dipole moment of 7.7 D per molecule when isolated and a total dipole of $11.6 \mathrm{D}$ per molecule when integrated in an infinite stack. Note that $\mu_{0}$ is the total dipole moment per amide, and not the axial component, which is why the permanent dipole moment per molecule is less than $3 \times 4=12 \mathrm{D}$. In terms of polarization this gives a total polarization $P_{\mathrm{s}}=48 \mathrm{mC} \mathrm{m}^{-2}$ for BTA-C6, which is slightly lower than the polarization found experimentally in Fig. 2(b). This corresponds to our earlier observation, where we found a higher than expected polarization which we attributed to an enhanced interaction between columns. ${ }^{23}$ For simplicity we will from here on only discuss the normalized polarization $P / P_{\mathrm{s}}$.

Second, there is a noticeable difference in shape of the hysteresis loops obtained by the simulation using the default parameters and the experiment in Fig. 2(a). The experimental loops are sharp and nearly rectangular, whereas the simulated loops are more slanted. To investigate possible causes for this discrepancy, Fig. 2(b) also shows the hysteresis loops obtained from a simulation without disorder, and one where the BTA columns have an enhanced separation distance. The effect of disorder and large column separations on the hysteresis loops has been investigated previously, ${ }^{25}$ and will be more extensively discussed further on in the text. For now, it suffices to conclude that the effect of disorder is too small to explain the discrepancy in slopes between experiment and simulations.

In contrast, the simulation with the increased column separation does show a sharp increase in the slope of the loop and has a general shape in agreement with the experiment. For the enhanced column separation there is essentially no interaction between columns. This suggests that in experiments, even with a small column separation, the interaction between columns is limited and we overestimate this interaction in the simulations. This contradicts our earlier suggestion that an enhanced interaction between columns is responsible for higher than expected polarizations. ${ }^{23}$ The reason for this overestimation could lie in the assumption of an isotropic dielectric medium with $\varepsilon_{\mathrm{r}}=2$ that surrounds the dipoles. In a real device this is obviously not the case, and the columns could be more strongly screened by for example the alkyl chains or regions of amorphous material.

Third, we analyze the coercive field, which is here defined as the field where the polarization is zero. Comparing the two loops in Fig. 2(a), the simulated coercive field is about four times higher than the experimental one - note the different $x$-axes for simulated and measured curves. This is often the case with simulations, where one usually obtains an intrinsic coercive field several orders of magnitude above the experimental value. ${ }^{1,26}$ The polarization switching in experiments is fully extrinsic and based on nucleation near defects, charged impurities, and/or interfaces. ${ }^{27}$ Evidently our model does not fully capture the complex dynamics of a real sample, even though it does include defects,

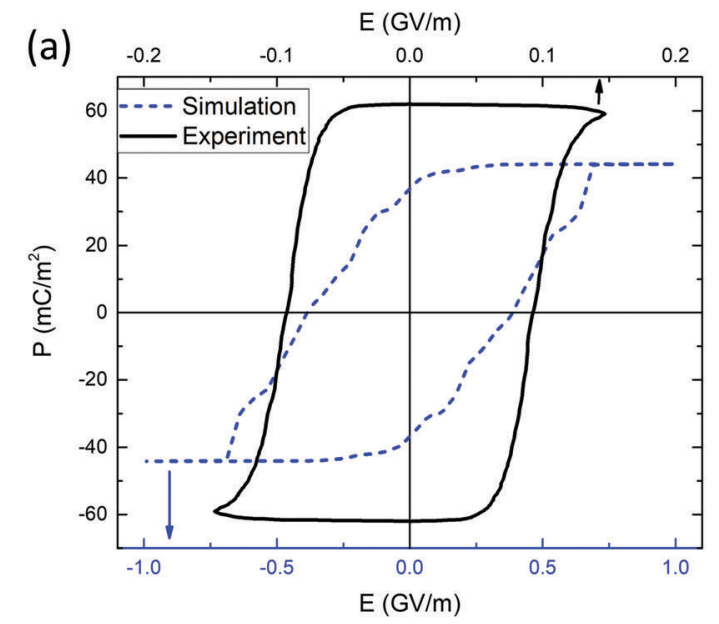

(b)

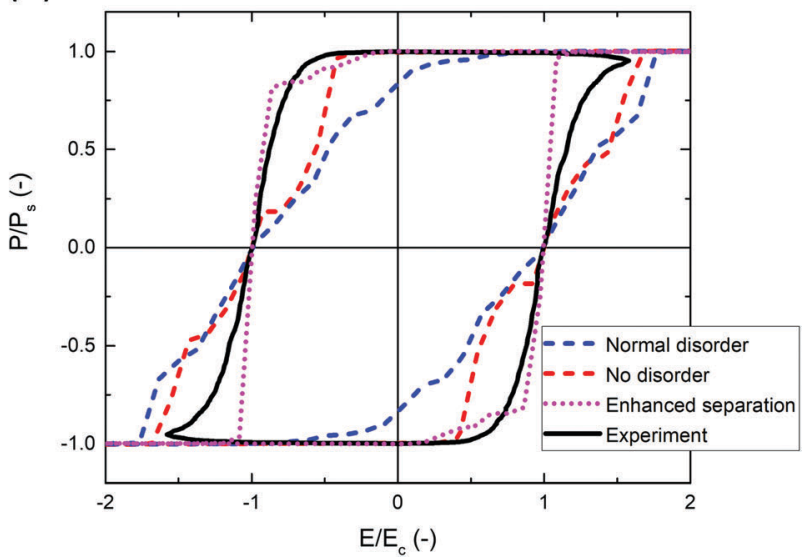

Fig. 2 (a) Typical simulated (blue) and experimental (black, taken from ref. 23) hysteresis loops for BTA. (b) Comparison of the normalized hysteresis loops of different simulations and the experiment. Enhanced separation corresponds to an intercolumnar distance $a=2 \mathrm{~nm}$, compared to the $a=1.67 \mathrm{~nm}$ used in all other simulations. Sweep frequency and sweep rate in simulations: $25 \mathrm{~Hz}, 100 \mathrm{GV} \mathrm{m}^{-1} \mathrm{~s}^{-1}$; experiment: $125 \mathrm{~Hz}, 75 \mathrm{GV} \mathrm{m}^{-1} \mathrm{~s}^{-1}$; all at $325 \mathrm{~K}$. 
disorder, and electrodes. A clear nucleation-limited behavior is seen (see ESI $\dagger$ video), with slow nucleation and subsequent fast growth of the switched domain and thereby should produce an extrinsic coercive field. However, considering the limited thickness of the simulation box, which is favorable for intrinsic switching, ${ }^{28-30}$ the simulated switching is likely still partly intrinsic. Simulations with an increased box size (40 $\mathrm{nm} v s .10 \mathrm{~nm})$ yielded, on the other hand, only a modest $(\sim 5 \%)$ reduction of the coercive field.

The coercive field is dependent on the field sweep speed and temperature. We can describe this dependency using the theory of thermally activated nucleation limited switching (TA-NLS) developed by Vopsaroiu et al. ${ }^{31}$ which gives for the coercive field:

$$
E_{\mathrm{c}}=\frac{W_{\mathrm{b}}-k_{\mathrm{B}} T \ln \left(\nu_{0} t / \ln (2)\right)}{V^{*} P_{\mathrm{s}}}
$$

where $W_{\mathrm{b}}=w_{\mathrm{b}} V^{*}$ is the flipping energy barrier, $k_{\mathrm{B}} T$ the thermal energy, $\nu_{0}$ an attempt frequency, $P_{\mathrm{s}}$ the saturation polarization, and $V^{*}$ is the nucleation volume. The waiting time $t$ is assumed to be inversely proportional to the field sweep frequency $f$. Previously we have shown that this TA-NLS theory provides a good description of the experimental switching kinetics in BTA. ${ }^{23-25}$

We verify the applicability of the TA-NLS model here by simulating hysteresis loops and determining the coercive field as a function of frequency and temperature. The results are shown in Fig. 3, together with the fit to the TA-NLS model. The attempt frequency is fixed at the input (phonon) frequency of $1 \mathrm{THz}$. The energy barrier $w_{\mathrm{b}}=0.18 \mathrm{eV} \mathrm{nm}^{-3}$ agrees with experimental values. The nucleation volume is $7.0 \mathrm{~nm}^{3}$, which is around 8 molecules and roughly corresponds to the average size of a subcolumn between defects.

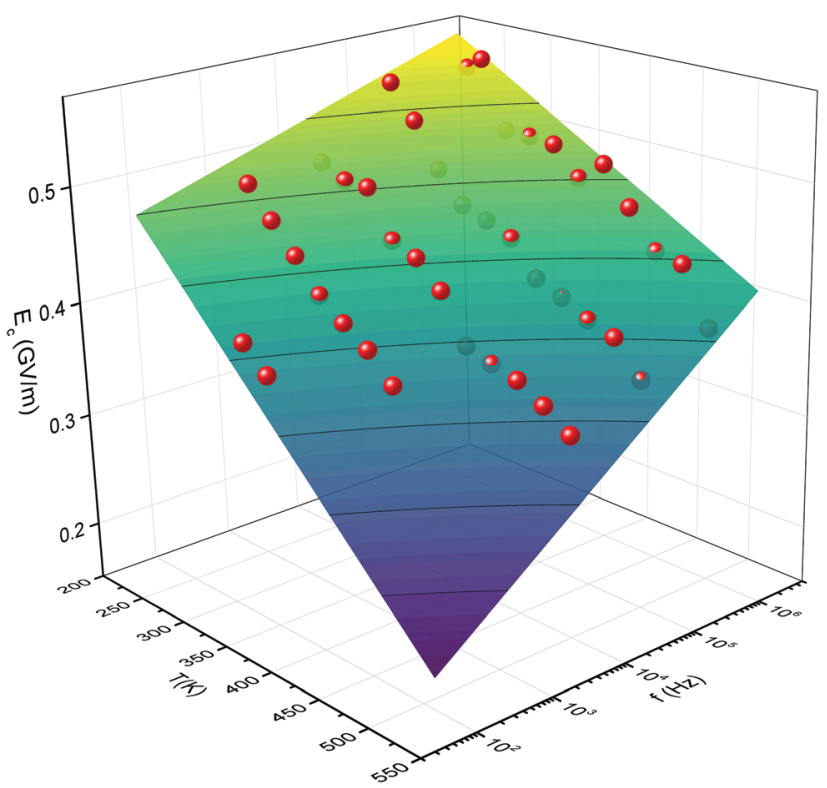

Fig. 3 Coercive field as a function of frequency and temperature calculated by the electrostatic KMC model (symbols). The surface is a fit to the data with eqn (1) with $w_{\mathrm{b}}=0.18 \mathrm{eV} \mathrm{nm}{ }^{-3}, V^{*}=7.03 \mathrm{~nm}^{3}$, and fixed $P_{\mathrm{s}}=48 \mathrm{mC} \mathrm{m}^{-2}$ and $\nu_{0}=1 \mathrm{THz}$.

\section{Retention}

An important but often overlooked property of ferroelectrics is their polarization retention. Especially in organic ferroelectrics the retention times are often poor which precludes practical applications. Several responsible driving forces for polarization loss have been discussed, such as the depolarization field caused by dead interface layers or imperfect screening by the electrodes. $^{24}$ The depolarization mechanism was previously argued to be R-relaxation, which is a collective reversal of the amide dipole moments in ferroelectric domains. ${ }^{15,24}$

Here, we investigate the retention by starting with a fully poled system and letting the polarization decay over time without an externally applied field. The resulting depolarization curve is generally described by a stretched exponential function:

$$
P=P_{0} \exp \left(-(t / \tau)^{b}\right)
$$

with $P_{0}$ the initial polarization, $b$ the stretching exponent, and $\tau$ the retention time.

Fitting the simulated depolarization curves in Fig. S5 (ESI $\dagger$ ) to eqn (2) gives the retention times shown in Fig. 4. A good agreement between simulations and experiment is seen when directly comparing the retention times. It should be noted that fitting the depolarization curves to eqn (2) is not trivial. Especially at the low temperatures that correspond to experimental conditions, where there is little polarization decay, the obtained retention times and stretch parameters can vary wildly. We therefore choose to fix the stretch parameter $b$ to 0.13 , an average value which gives decent fits for all temperatures (see Fig. S5, ESI $\dagger$ ). For experimental depolarization curves the stretch parameter is usually found to be around 0.5 . $^{24}$ The reasons for this difference are unclear at present but might relate to the absence of dead layers in the simulation.

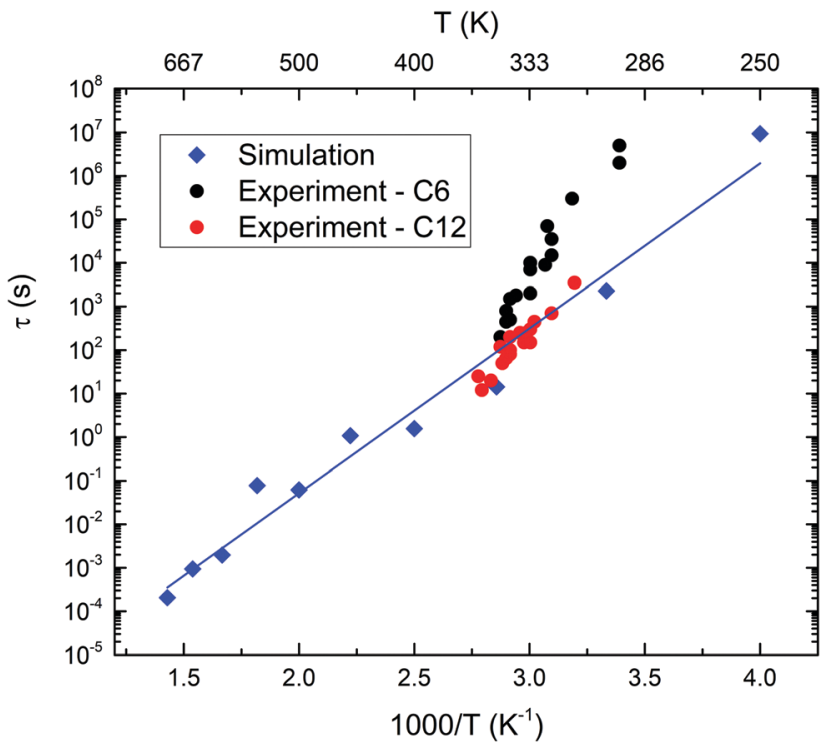

Fig. 4 Arrhenius plot showing the polarization retention time $\tau$ versus the inverse temperature. Retention times are obtained by fitting eqn (2) to the simulation results with a fixed parameter $b=0.13$. The blue line is a fit of the simulation data to eqn (3) with $\nu_{0}=0.38 \mathrm{MHz}$ and $W_{\mathrm{b}}=0.75 \mathrm{eV}$. Experimental data for BTA-C6 and $\mathrm{C} 12$ are taken from ref. 23. 
As was done for the coercive field, we use the TA-NLS model to describe the depolarization by thermal activation over an energy barrier $W_{\mathrm{b}}{ }^{31}$ The retention time $\tau$ is then given by

$$
\tau=\frac{1}{2 \nu_{0}} \exp \left(W_{\mathrm{b}} / k_{\mathrm{B}} T\right)
$$

Fitting the results to eqn (3) gives $\nu_{0}=0.38 \mathrm{MHz}$ and $W_{\mathrm{b}}=0.75 \mathrm{eV}$. This depolarization activation energy is similar to what is found experimentally. ${ }^{23,24}$ However, the attempt frequency differs orders of magnitude from the input attempt frequency $\nu_{0}$ that was recovered in the analysis of the switching kinetics. A similar deviation between the attempt frequencies found in analyzing switching and depolarization kinetics is also observed in experiments. ${ }^{23} \mathrm{We}$ interpret this to indicate that for a stable nucleus to form, a single dipole flip is not enough. Instead, multiple flips need to occur simultaneously, leading to a reduced attempt frequency. This will be further discussed below in the section on the effect of disorder.

\section{Flipping modes and the 2:1 state}

So far, we have assumed that when a dipole flips, only its $z$-component changes. However, another mode of flipping is possible as well, corresponding to a full inversion of the dipole vector. Both flipping modes are illustrated in Fig. 5(a) and will be called $z$-flip and full flip from here on. Fig. 5(a) shows that the $z$-flip mode changes the helicity of the triple helix, whereas the full flip mode does not. Flipping of the helicity upon polarization reversal has consequences when investigating switching on BTA substituted with enantiomerically pure chains, which will be discussed in more detail in a future work.

We can artificially restrict the system to one of the modes and simulate the hysteresis loops. We find that the coercive field of the full flip mode is $\sim 4$ times higher than that of the $z$-flip mode, see Fig. 5(b). The $z$-flip mode is thus energetically favored and should be the one responsible for dipole flipping in experiments. We can also analyze the energetics of the full flip mode by determining the coercive field as a function of temperature and frequency, as was done in Fig. 3 for the $z$-flip mode. We find (see Fig. S6, ESI $\dagger$ ) an energy barrier of $0.78 \mathrm{eV} \mathrm{nm}^{-3}$ and a nucleation volume of 3 dipoles, which is significantly higher respectively lower than for the $z$-flip. The high energy barrier is caused by the unfavorable head-to-head interaction that occurs between neighbors when a single dipole is fully flipped. The smaller nucleation volume is because in the full flip mode only one helix will switch at a time, whereas in the $z$-flip mode all three helices must flip nearly simultaneously.

The hysteresis loop of the full flip mode as obtained with $\mathrm{kMC}$ is not perfectly square and shows shoulders at $\pm 2.5 \mathrm{GV} \mathrm{m}^{-1}$. This shoulder corresponds to an intermediate state where in each column the dipoles in one helix are pointing in opposite direction to the other two helices, as shown in Fig. 5(a) and Fig. S7 (ESI $\dagger$ ). This state is incompatible with the $z$-flip mode, because there switching occurs by breaking the whole triple helix structure molecule by molecule, instead of just reversing one helix at a time. The shoulder is therefore not observed in the $z$-flip hysteresis loop. The fact that it is neither observed in experiments is further evidence that in reality, flipping occurs through the $z$-flip mode. This is a refinement of our previous conclusion where we tacitly assumed that R-relaxation, which is the collective reversal of a domain that is responsible for the polarization loss, would correspond to the full flip mode. ${ }^{15,24}$

The stability of this so-called 2:1 state has been investigated previously with DFT and MD, and it was found that for zero applied field this state is energetically more favorable than the 3:0 state with all dipoles pointing in one direction. ${ }^{19,20,32}$ This is mainly due to the electrostatic interaction that favors antiparallel alignment of the three dipole helices. The fact that the 2:1 state is observed as an intermediate in the simulated hysteresis loops confirms that it indeed corresponds to a (local) energy minimum. A more detailed analysis of the energetics of this state can be found in the ESI. $\dagger$

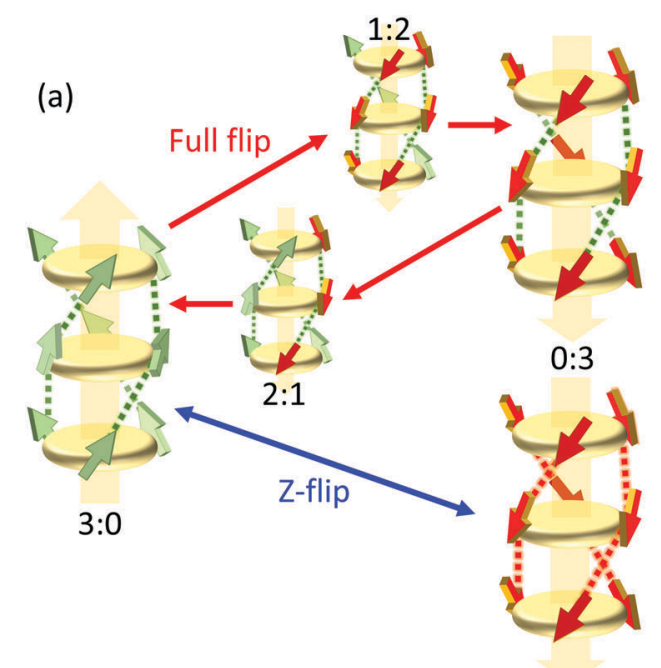

(b)

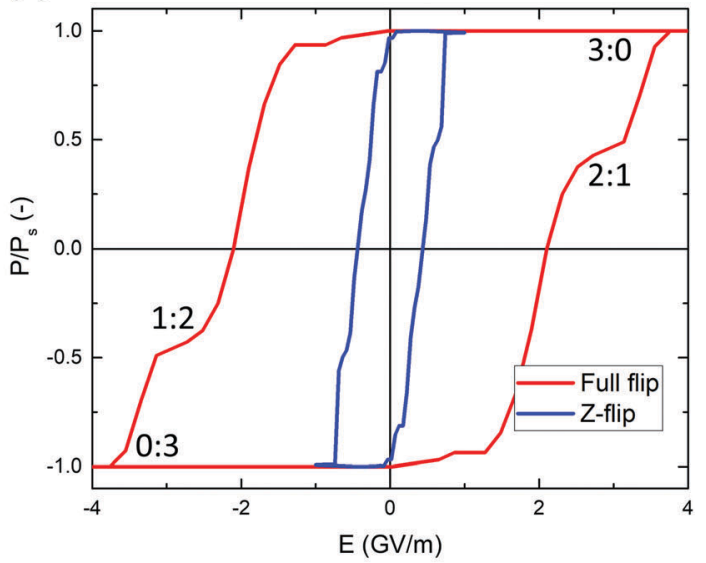

Fig. 5 (a) Schematic of the two flipping modes. For the full flip mode, the intermediate 1:2 and 2:1 states are also shown. (b) Hysteresis loops for both modes at $300 \mathrm{~K}$ and $250 \mathrm{~Hz}$ obtained by KMC. The apparent shoulders in the $z$-flip curve are an effect of the discrete field sweep steps, and do not correspond to a specific state as in the full flip curve. 
The fact that the $z$-flip mode is the dominant flipping mode has been demonstrated previously through MD simulations by Bejagam et $a .^{18}$ In these simulations, both modes of flipping are allowed. The authors start with a BTA column polarized in one direction and apply an electric field to reverse the polarization. They found that upon polarization reversal, the helicity of the triple helix also reverses, corresponding to the $z$-flip mode. This behavior was attributed to a combination of electrostatic and steric effects. ${ }^{33}$ Our current results show that the $z$-flip mode is favored even when steric effects are ignored.

We have investigated the flipping process with further MD simulations (see Fig. S10 and ESI $\dagger$ for a detailed description and analysis of the MD simulations). We again find that the $z$-flip mode is the dominant mode, although the differences with the full flip mode are small. In the full flip mode, investigated here by examining transitions from the 2:1 state, a variety of intermediate states is possible due to thermal fluctuations. Indeed, when we apply a low electric field of $0.28 \mathrm{GV} \mathrm{m}^{-1}$ on the 2:1 state, we observe small fluctuations, i.e. one of the BTA residues in the stack twists in the $x y$-plane resulting in an interchange of the values of two dihedral angles while keeping the same helicity, since this electric field is not high enough to induce the full flip in the time of our simulation. When a higher field of $0.36 \mathrm{GV} \mathrm{m}^{-1}$ is applied on the same 2:1 stack, both $M$ - and $P$-helicities are observed. Some intermediate states do not even keep the favorable H-bonding network, and the H-bonding helix is disrupted for a short time. With increasing value of the electric field, the self-assembled stack is fully switched from $2: 1$ to $0: 3$. On the other hand, when we apply an electric field of $0.22 \mathrm{GV} \mathrm{m}^{-1}$ on a fully polarized 3:0 state, the dihedral angles flip through a value of $0^{\circ}$ and consequently the helicity changes, without going through any intermediate states. This $z$-flip is therefore energetically more favorable since it requires a lower electric field than the full flip.

\section{The effect of disorder}

In our previous experimental work we have observed that the morphology and more specifically the degree of disorder can have a major influence on the ferroelectric properties of BTA. $^{23,25}$ Therefore we will now study the effects of disorder on the simulated hysteresis loops and retention. We control the disorder in the system by changing the average length of the subcolumns between defects. At each defect there is a translational shift and randomization of the rotational angle, as discussed before and shown in Fig. S3 (ESI $\dagger$ ). We consider four different cases: high, medium, low and no disorder, corresponding to a mean subcolumn length of $7,15,20$, and infinite molecules, respectively. Note that all results reported above were obtained for the system with medium disorder.

Comparing the hysteresis loops for high, medium and no disorder in Fig. 6(a) shows that there is only a very minor decrease in the coercive field upon increasing the disorder. This holds for the whole range of frequencies and temperatures as shown in Fig. S11 (ESI $\dagger$ ). The change in shape of the loops is more significant, with more slanted loops for higher disorders. To explain this, we consider the hysteresis loop of our system as the sum of the response of the subcolumns. This is the idea behind the Preisach theory, which considers a ferroelectric to be a collection of perfect hysterons. ${ }^{34}$ In this case, each subcolumn can be seen as such a hysteron with a square hysteresis loop and a well-defined coercive field. Due to the disorder in the system, there is a distribution in these coercive fields, which causes the total loop to become slanted. The higher the disorder, the broader the distribution in coercive fields, and the more slanted the loop becomes. A similar effect occurs when the distance between columns is increased as in Fig. 2(b), because there will no longer be any interaction between columns, as previously shown. ${ }^{25}$

In contrast to the influence on the hysteresis loops, the influence of disorder on the polarization retention is significant. Fig. 6(b) shows the retention times as determined by the procedure described earlier. The retention is increased by several orders of magnitude upon decreasing the disorder. In the case of no disorder no polarization loss is observed at all at lower temperatures.

The difference between the disorder dependence of the coercive field and retention stems from differences in the nucleation mechanism. By visual inspection of the simulation results we identified the typical nuclei for both cases as shown in Fig. 6(c and d). We find that for field-driven polarization reversal, nucleation almost exclusively occurs at the electrodes, and a nucleus of about three dipoles is required before a subcolumn fully switches. For spontaneous polarization reversal on the other hand, nucleation occurs mostly at defects in the bulk of the material. The nucleus is also slightly larger, which supports our earlier speculation that the reduced attempt frequency for depolarization is caused by a larger nucleus, involving a higher-order coincidence. Further inspection of the energetics of the nucleation processes reveals the reason for these different locations of the nuclei. Nucleation at an electrode is generally favored, but unstable without an applied electric field. In the case of the spontaneous reversal, nucleation therefore has to occur at defects, where it is stable without applied field. A more detailed explanation can be found in the ESI. $\dagger$ The disorder has thus little influence on the hysteresis loops as nucleation anyhow occurs at the electrodes, whereas it heavily affects the retention where nucleation occurs at defects.

This observation provides a way to increase performance of (organic) ferroelectric devices. For application purposes a high retention time is desired, whereas the coercive field should still be low enough to allow reasonable operating voltages. In typical (inorganic) ferroelectrics the retention time and coercive field are usually coupled; when one increases, the other increases as well, as both scale with the energy barrier for switching. ${ }^{23}$ Our results show that the additional degrees of freedom offered by supramolecular ferroelectrics allow tailoring of the disorder. On the one hand, improving processing and changing e.g. side chains to promote stacking might be the key to get high retention times while maintaining reasonable coercive fields. ${ }^{23}$ On the other hand, increasing disorder might facilitate devices with very fast response times that can be operated at high frequencies.

\section{Ground state}

We can investigate the ground state of our system by letting a simulation without applied field run until an equilibrium is reached. 
(a)

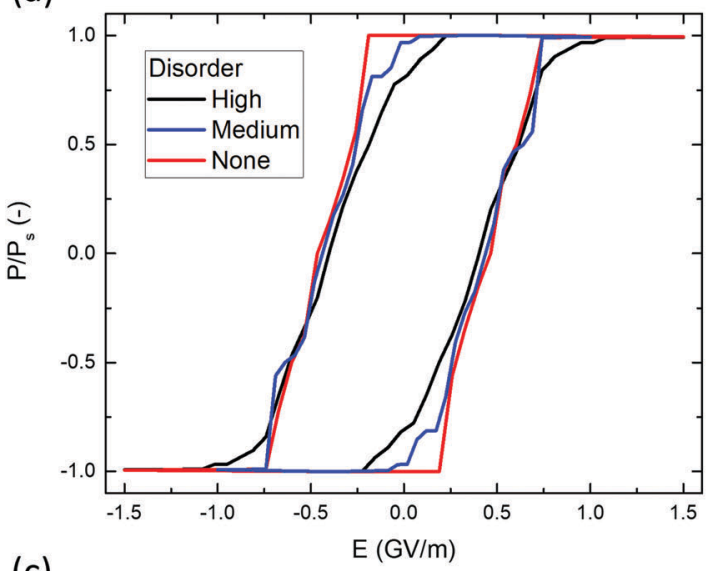

(c)

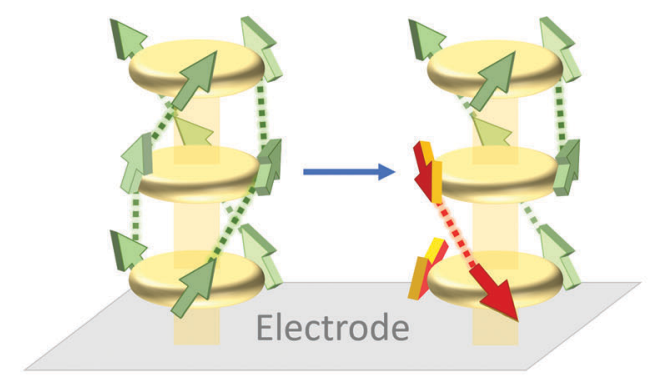

(b)

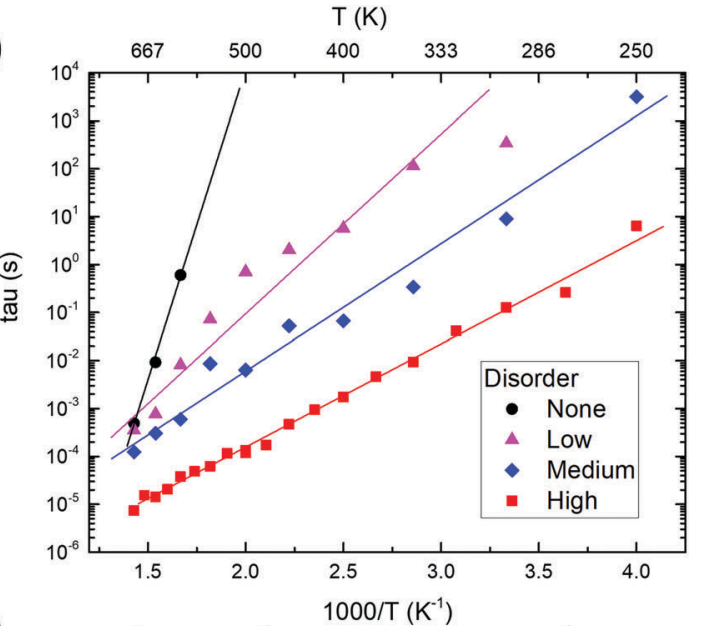

(d)

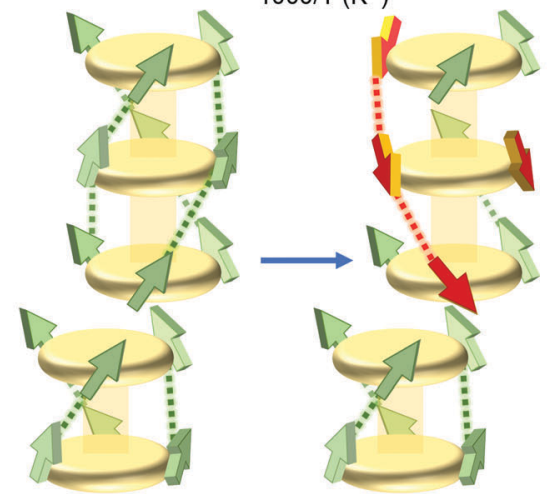

Fig. 6 The effect of disorder on (a) the hysteresis loop and (b) the retention time. The hysteresis loops were simulated for $250 \mathrm{~Hz}$ and $300 \mathrm{~K}$. Retention times were obtained by fitting the depolarization curves to eqn (2) with a fixed stretching parameter $b=0.2$. No polarization loss was observed after 1 ms for the no disorder case below $600 \mathrm{~K}$. Solid lines are a guide to the eye. The two nucleation mechanisms at an electrode (c) and defect (d) correspond to the field-driven and spontaneous polarization reversal, respectively.

Details on these simulations are presented in the ESI. $\dagger$ When no disorder is present, we find that the ground state is a mixture of up and down fully polarized columns with zero net polarization. We can thus represent the domain structure as the $2 \mathrm{D}$ top view in Fig. 7. A stripe-like domain structure is observed, which is typical for a frustrated antiferroelectric. The electrostatic interaction between the macrodipoles of two neighboring columns tends to align them anti-parallel. Due to the geometric frustration the ground state is highly degenerate, and complex domain structures can be formed.

We can quantitatively characterize the domain structure by looking at the correlation coefficients, which are defined as the dot product of a dipole and the mean of its (next) nearest neighbors (eqn S6, ESI $\dagger$ ). The correlation coefficients for the domain structure in Fig. 7 are shown in Fig. S13 (ESI $\dagger$ ). We find that indeed nearest neighbors tend to be antiparallel, and consequently next nearest neighbors have a slight tendency to be parallel.

When disorder is introduced into the system, columns are no longer necessarily fully polarized, shown in Fig. S14 (ESI †). Within a column, subcolumns can have different polarizations, resulting in partially polarized columns and a more complicated three-dimensional domain structure. The correlation coefficients are now shifted towards zero (no correlation), indicating a decrease

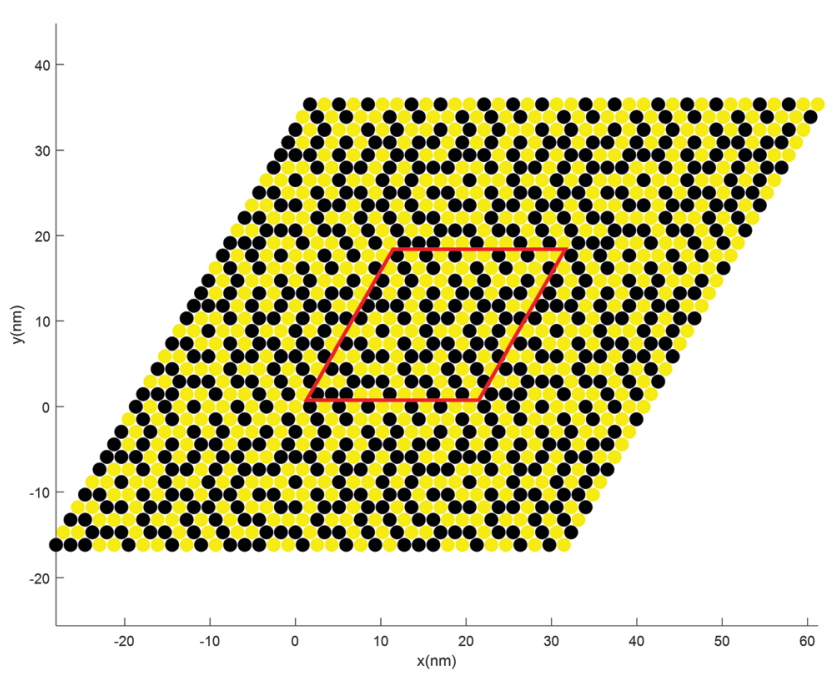

Fig. 7 The ground state domain structure obtained after full depolarization of a system without disorder. To elucidate the domain structure, a periodic repetition of the original grid (indicated in red) is shown.

in the tendency of (next nearest) neighbors to align antiparallel (parallel) and thus a more disordered domain structure.

A similar antiferroelectric domain structure was previously suggested for BTA crystals based on X-ray diffraction experi- 
ments. ${ }^{21,35}$ Zehe et al. studied several BTA compounds with different side-chains to investigate the interplay between electrostatic and steric interactions. They found that compounds with bulky side-chains, and thus larger steric hindrance, can form mesoscale domains with a net polarization. Compounds with smaller side-chains, such as the BTA-C6 investigated here, only formed non-polarized domain structures as in Fig. 7 due to the dominating antiferroelectric electrostatic interaction. The authors support their conclusions with a simple 2D Ising model based on two interaction constants for the nearest neighbor and next nearest neighbor interaction where the latter are associated with steric interactions and can cause preferential parallel alignment when of correct sign and magnitude. However, the relation of the interaction constants to actual materials remains somewhat unclear since it seems unlikely that steric interactions play a significant role for anything but the nearest neighbor interactions.

It should be noted that our model only accounts for electrostatic interactions and ignores steric effects beyond the attemptto-flip frequency $\nu_{0}$. Nevertheless, we obtain a good agreement with experiments. From this we can conclude that steric effects are of minor importance in the case of BTA-C6, in agreement with the conclusion from Zehe et al. Steric effects could play a role for other polar columnar liquid crystals of bulkier molecules, ${ }^{36}$ where the ground state will still be antiferroelectric, but where the steric effects might allow mesoscale polar domains.

Even though the ground state of BTA is thus antiferroelectric, this has little relevance for its practical applications as a ferroelectric. As we have shown here and in experiments previously, it is possible to polarize the material completely by applying an electric field. It is then kinetically frozen in this state due to the high activation energy for switching. Depending on the disorder, retention times of several months and more can be obtained. ${ }^{23,37}$ The material will thus 'never' reach its ground state once it has been polarized.

\section{Conclusions}

In summary, we have developed an electrostatic model that is used as basis for 3D kinetic Monte Carlo simulations to describe switching kinetics in ferroelectrics. We found good agreement between simulations and experiments for a prototype molecular organic ferroelectric. Since the model does not explicitly include steric effects, this leads to the conclusion that these must be of minor importance for this material. Both hysteresis loops and depolarization curves could be simulated for a large range of temperatures and timescales. We investigated different flipping modes and found that the results of our model agree with those from molecular dynamics simulations. The theory of thermally activated nucleation limited switching was used to analyze all results and gave an energy barrier for switching and depolarization of around $1 \mathrm{eV}$. Even though the ground state of the system is found to be antiferroelectric, this state is under practical conditions never reached due to the slow kinetics associated with this high energy barrier.
Finally, we found that nucleation occurs differently in the case of spontaneous polarization reversal (depolarization) compared to field-driven reversal in a hysteresis loop. During depolarization nucleation occurs at defects caused by disorder, while during a hysteresis loops it occurs at the electrodes. By reducing the disorder, the retention time can thus be dramatically increased while the coercive field remains unchanged. This provides a new pathway for the rational design and optimization of ferroelectric devices, specifically for memory applications.

Although these results are obtained for a specific material, all conclusions are applicable to the whole class of columnar organic ferroelectrics. More generally, the model itself could be adapted to study other ferroelectric systems, such as BTO or PVDF. ${ }^{8,38}$ For inorganic ferroelectrics however, one should take into account an elastic term that penalizes domain wall formation, which will increase the computational complexity. Since the model works for any fixed morphology, including irregular ones, systems with extended disorder, such as dipolar glasses, could also be investigated although any increase in the degrees of freedom will come at the cost of increased computation times. Finally, the insight into the difference between field-driven and spontaneous reversal kinetics, brought about by differences in the rate-limiting nucleation site, can be expected to be relevant for disordered ferroelectrics in general.

\section{Conflicts of interest}

The authors declare no competing financial interests.

\section{Acknowledgements}

T. D. C. acknowledges financial support from the Swedish Government Strategic Research Area in Materials Science on Functional Materials at Linköping University (Faculty Grant SFO Mat LiU no. 2009 00971). I. U. and P. N. acknowledge funding by Vetenskapsrådet. M. L. thanks SeRC (Swedish e-Science Research Center) for funding. The Swedish National Infrastructure for Computing (SNIC) at National Supercomputer Centre (NSC) and Center for High Performance Computing (PDC) are acknowledged for providing computer resources.

\section{References}

1 J. Liu, W. Chen, B. Wang and Y. Zheng, Materials, 2014, 7, 6502-6568.

2 T. Shimada, S. Tomoda and T. Kitamura, Phys. Rev. B: Condens. Matter Mater. Phys., 2010, 81, 144116.

3 J. Junquera and P. Ghosez, Nature, 2003, 422, 506-509.

4 N. Sai, A. M. Kolpak and A. M. Rappe, Phys. Rev. B: Condens. Matter Mater. Phys., 2005, 72, 020101.

5 R. E. Cohen and H. Krakauer, Phys. Rev. B: Condens. Matter Mater. Phys., 1990, 42, 6416-6423.

6 T. Nishimatsu, U. V. Waghmare, Y. Kawazoe and D. Vanderbilt, Phys. Rev. B: Condens. Matter Mater. Phys., 2008, 78, 104104.

7 V. Boddu, F. Endres and P. Steinmann, Sci. Rep., 2017, 7, 806. 
8 M. Kühn and H. Kliem, Ferroelectrics, 2008, 370, 207-218.

9 B. G. Potter, V. Tikare and B. A. Tuttle, J. Appl. Phys., 2000, 87, 4415-4424.

10 T. K. Bose and J. Saha, Phys. Rev. Lett., 2013, 110, 265701.

11 S. Horiuchi and Y. Tokura, Nat. Mater., 2008, 7, 357-366.

12 H. Takezoe and F. Araoka, Liq. Cryst., 2014, 41, 393-401.

13 S. Cantekin, T. F. A. de Greef and A. R. A. Palmans, Chem. Soc. Rev., 2012, 41, 6125-6137.

14 A. V. Gorbunov, T. Putzeys, I. Urbanavičiūtè, R. A. J. Janssen, M. Wübbenhorst, R. P. Sijbesma and M. Kemerink, Phys. Chem. Chem. Phys., 2016, 18, 23663-23672.

15 C. F. C. Fitié, W. S. C. Roelofs, P. C. M. M. Magusin, M. Wübbenhorst, M. Kemerink and R. P. Sijbesma, J. Phys. Chem. B, 2012, 116, 3928-3937.

16 C. F. C. Fitié, W. S. C. Roelofs, M. Kemerink and R. P. Sijbesma, J. Am. Chem. Soc., 2010, 132, 6892-6893.

17 C. Kulkarni, S. K. Reddy, S. J. George and S. Balasubramanian, Chem. Phys. Lett., 2011, 515, 226-230.

18 K. K. Bejagam, C. Kulkarni, S. J. George and S. Balasubramanian, Chem. Commun., 2015, 51, 16049-16052.

19 K. K. Bejagam, G. Fiorin, M. L. L. Klein and S. Balasubramanian, J. Phys. Chem. B, 2014, 118, 5218-5228.

20 R. Q. Albuquerque, A. Timme, R. Kress, J. Senker and H. W. Schmidt, Chem. - Eur. J., 2013, 19, 1647-1657.

21 C. S. Zehe, J. A. Hill, N. P. Funnell, K. Kreger, K. P. Van der Zwan, A. L. Goodwin, H. W. Schmidt and J. Senker, Angew. Chem., Int. Ed., 2017, 56, 4432-4437.

22 C. J. Fennell and J. D. Gezelter, J. Chem. Phys., 2006, 124, 234104.

23 I. Urbanavičiūtè, X. Meng, T. D. Cornelissen, A. V. Gorbunov, S. Bhattacharjee, R. P. Sijbesma and M. Kemerink, Adv. Electron. Mater., 2017, 1600530.
24 A. V. Gorbunov, X. Meng, I. Urbanavičiūtè, T. Putzeys, M. Wübbenhorst, R. P. Sijbesma and M. Kemerink, Phys. Chem. Chem. Phys., 2017, 19, 3192-3200.

25 I. Urbanavičiūtè, T. D. Cornelissen, X. Meng, R. P. Sijbesma and M. Kemerink, Nat. Commun., 2018, 9, 4409.

26 G. Gerra, A. K. Tagantsev and N. Setter, Phys. Rev. Lett., 2005, 94, 107602.

27 S. V. Kalinin, A. N. Morozovska, L. Q. Chen and B. J. Rodriguez, Rep. Prog. Phys., 2010, 73, 056502.

28 S. Ducharme, V. M. Fridkin, A. V. Bune, S. P. Palto, L. M. Blinov, N. N. Petukhova and S. G. Yudin, Phys. Rev. Lett., 2000, 84, 175-178.

29 P. Chandra, M. Dawber, P. B. Littlewood and J. F. Scott, Ferroelectrics, 2004, 313, 7-13.

30 J. Y. Jo, Y. S. Kim, T. W. Noh, J. G. Yoon and T. K. Song, Appl. Phys. Lett., 2006, 89, 232909.

31 M. Vopsaroiu, J. Blackburn, M. G. Cain and P. M. Weaver, Phys. Rev. B: Condens. Matter Mater. Phys., 2010, 82, 024109.

32 K. K. Bejagam and S. Balasubramanian, J. Phys. Chem. B, 2015, 119, 5738-5746.

33 D. B. Korlepara, K. K. Bejagam and S. Balasubramanian, J. Phys. Chem. B, 2017, 121, 11492-11503.

34 F. Preisach, Z. Phys., 1935, 94, 277-302.

35 A. Simonov, T. Weber and W. Steurer, J. Appl. Crystallogr., 2014, 47, 2011-2018.

36 D. Miyajima, F. Araoka, H. Takezoe, J. Kim, K. Kato, M. Takata and T. Aida, Science, 2012, 336, 209-213.

37 I. Urbanavičiūtè, S. Bhattacharjee, M. Biler, J. A. M. Lugger, T. D. Cornelissen, P. Norman, M. Linares, R. P. Sijbesma and M. Kemerink, Phys. Chem. Chem. Phys., under review.

38 A. Leschhorn, S. Djoumbou and H. Kliem, J. Appl. Phys., 2014, 115, 114106. 